\title{
Optimization of Gas Transmission Pipeline using Ant Colony Optimization
}

\author{
Akshat Sharma \\ School of Computer Science \\ UPES Dehradun
}

\author{
Nitya Chauhan \\ School of Computer Science \\ UPES Dehradun
}

\author{
Aabhay Bhardwaj \\ School of Computer Science \\ UPES Dehradun
}

\author{
Prakhar Tiwari \\ School of Computer Science \\ UPES Dehradun
}

\author{
Nitin Arora \\ Electronics \& Computer Discipline \\ IIT Roorkee
}

\begin{abstract}
Presently, gas is one of the most important natural resources available on Earth. Gas is required in many fields. Gas flow from one source to the destination using pipes. Flowing is gas through pipes sometime can be very risky. For, the flow of gas through piles one should follow the smallest path as per the distance. But, not only the distance one should take care of the pressure as well. Pressure at different points also plays an important role in the flow of gas. Optimizing the pipeline route plays an important role in making the transportation eco-friendly, efficient and safe. As per the literature, the flow of gas is done using brute force algorithms (Complexity$\mathrm{O}(|\mathrm{V}|)$ ), Dijkstra Algorithm (Complexity-O $|\mathrm{V}|^{2}$ ), Dynamic Programming Algorithms (Complexity-O(|V| $\left.\left.\right|^{2} 2^{|\mathrm{V}|}\right)$ ), where, $|\mathrm{V}|$ is representing the number of nodes. In this paper, Traveling Salesman Problem is being solved using Ant Colony Optimization Algorithm taking pressure and distances for the flow of gas from one point to another point.
\end{abstract}

\section{Keywords}

Pressure, Distance, Inclination, Power, Ant Colony Optimization

\section{INTRODUCTION}

"Not all heroes wear capes" and the perfect example to support the phrase is the PIPELINE SYSTEM. We cannot imagine our everyday life without the pipeline system because they support the movement of water, gas, oil, sewer hence the length of Gas Pipelines was $10772 \mathrm{Kms}$ as on $31^{\text {st }}$ March 2011 and the U.S. has the world's largest network of pipelines in the world. America depends on a network of more than 207,800 miles of liquids pipelines, over 300,000 miles of gas transmission pipelines, and more than 2.1 million miles of gas distribution pipelines [3]. Pipelines provide an efficient means to transport crude oil, hydrocarbon products and gas, liquefied petroleum gas and other raw materials and products quickly, efficiently and safely. However, this vital element of Gas infrastructure needs to be permanently and efficiently monitored in order to ensure safe transportation, prevent damages from intrusion, achieve minimum downtime and life cycle costs, and to maintain environmental and quality standards. When it comes to things that make the pipeline operators concerns about are namely, the delivery amount and economic benefit. The aim of the pipeline operation optimization is to reach the maximum gas delivery amount, to reach the maximum economic benefit, or to reach two or more objectives simultaneously. Since the late 1960s, Dynamic Programming has been the go to solution technique to solve any pipeline optimization problem. It's fast computational speed on sequential systems and it's insensitivity to simulation non-linearity, it became a major part of the way pipeline systems work today. But with great advantages comes two major disadvantages: Dynamic Programming is applicable to only non-cyclic networks (linear) and The computation cost increases exponentially when the dimensions of the problem increase. Hence, the Dynamic Programming is difficult to be extended to large-scale and complicated pipeline networks involving hundreds of gas sources, consumers, and pipes. Ford Fulkerson algorithm can also be used for calculating the maximum flow in the networks. Modified ford fulkerson algorithm is proposed in [7-11]

Ant Colony Optimization technique is a newer evolutionary method and was inspired by the behavior of ants where they search for the shortest route between food and their home following a chemical called "Pheromone". The application of ant colony has been used for minimizing fuel cost in compressors. Ant colony optimization can also be used in mobile ad-hoc networks [6]. Ant Colony Optimization wins over classical methods because of its simple concept, ease to merge with other methods for better optimization and its capability to solve problems with no initial solution.

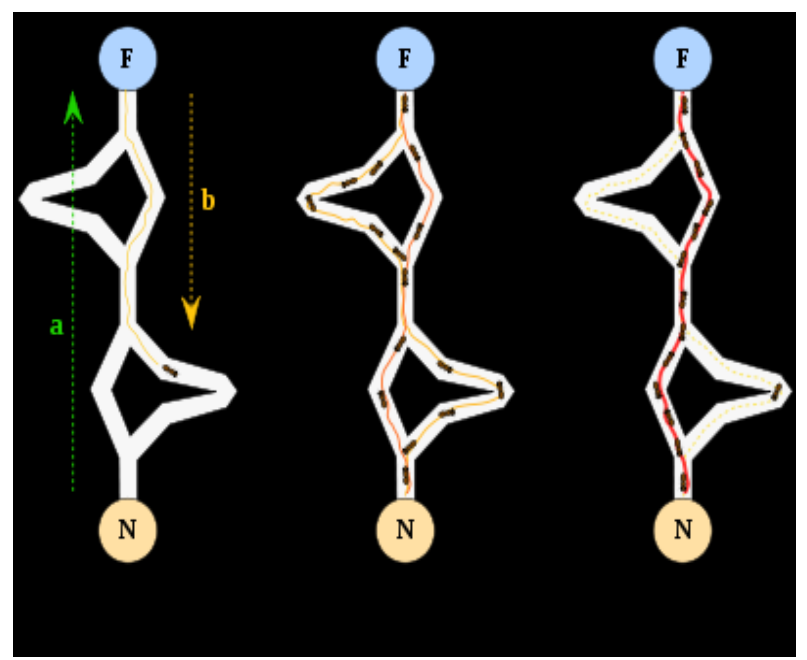

Fig. 1: Flowing of Ants in

(a) Ants start with making a pheromone trail. (b) Some ants choose the left path, some choose the right path and some choose the middle path. The choice is random. Since ants move at approximately a constant speed, the ants which choose the right, shorter, path reach the opposite decision point faster than those which choose the left, longer path. (c) 
Pheromone accumulates at a higher rate on the shorter path. The intensity of red lines is directly proportional to the amount of pheromone deposited by the ants. Considering Fig1, ants arrive at a decision point in which they have to decide which path to opt for. Since they have no clue about which is the best choice, they choose the path very randomly. It can be expected that, on average, half of the ants decide to turn left and the other half to turn right. After a short transitory period the difference in the amount of pheromone on the two paths is sufficiently large so as to influence the decision of new ants coming into the system. From now on, new ants will prefer in probability to choose the middle path. This in turn increases, with a positive feedback effect, the number of ants choosing the middle, and shorter path. Very soon all ants will be using the shorter path. The main idea is that of having a set of agents, called ants (hypothetical), search in parallel for good solutions to the TSP and cooperate through pheromone mediated indirect and global communication through the nodes (refineries) and synergistic use of cooperation among many relatively simple agents which communicate by distributed memory implemented as pheromone deposited on edges of a graph. Informally, ant system works as follows. Each ant generates a complete tour by choosing the cities according to a probabilistic state transition rule; ants prefer to move to cities which are connected by short edges with a high amount of pheromone. Once all ants have completed their tours a fraction of the pheromone evaporates on all edges (edges that are not refreshed become less desirable), the process is then iterated.

\subsection{Motivation behind the work}

Pipeline route configuration is an essential assignment for the oil and gas industry, and the route chosen can fundamentally influence the success or failure of a task. Subsequently, it is important to plan pipeline routes to be Eco-friendly, efficient and safe. Obstacle avoidance is one of the principle issues that influence pipeline route determination. In this, we propose a strategy for planning a programmed shortest route. The Ant colony optimization was utilized to make consequently created pipeline routes. The calculations were quick and the strategy was demonstrated to be viable and simple to use. Based on this we figure out the following problems:
1. Gas transmission through pipeline can become a problem in difficult terrains which can increase the cost exponentially. Additionally laying the pipelines can put an unsafe impact on Environment coming in the way of pipelines.

2. Problem is to minimize the cost factor in Gas transportation through pipeline.

Rest of the paper is divided as follows: Section 2 gives a brief idea about literature part. Methodology used and results has been described in section 3. Section 4 contains conclusion and future scope.

\section{RELATED WORK}

Due to the amount of complexity and diversity, it is pretty difficult to deduce the optimization design of gas pipeline network layout. The optimization techniques such as the Dynamic Programming method were in use for such complex tasks. Some of the related works are: Baeza et al. [1] used this technique for solving this problem. A comparison between ACO and Dijkstra algorithms for optimal ore concentrate pipeline routing. Soeiro Ferreira et al. [2] Optimization of a pump-pipe system by dynamic programming. Engineering Optimization - ENG OPTIMIZ. 7. 241-251. $10.1080 / 03052158408960641$. The way in which this task was done traditionally is a time-consuming and computationally difficult process. Therefore, the intelligent optimization techniques with good global search ability and robustness have drawn extensive attention and widely used for it.

\section{METHODOLOGY}

Due to the fact that only less technical risks can be afforded and the availability of a short time period, Rapid Application Development (RAD) is the best suited methodology to be applied. In the RAD model, the functional modules are developed in parallel as prototypes and are integrated to make the complete product for faster product delivery. Since there is no detailed pre-planning, it makes it easier to incorporate the changes within the development process.[4]

\subsection{Flowchart}

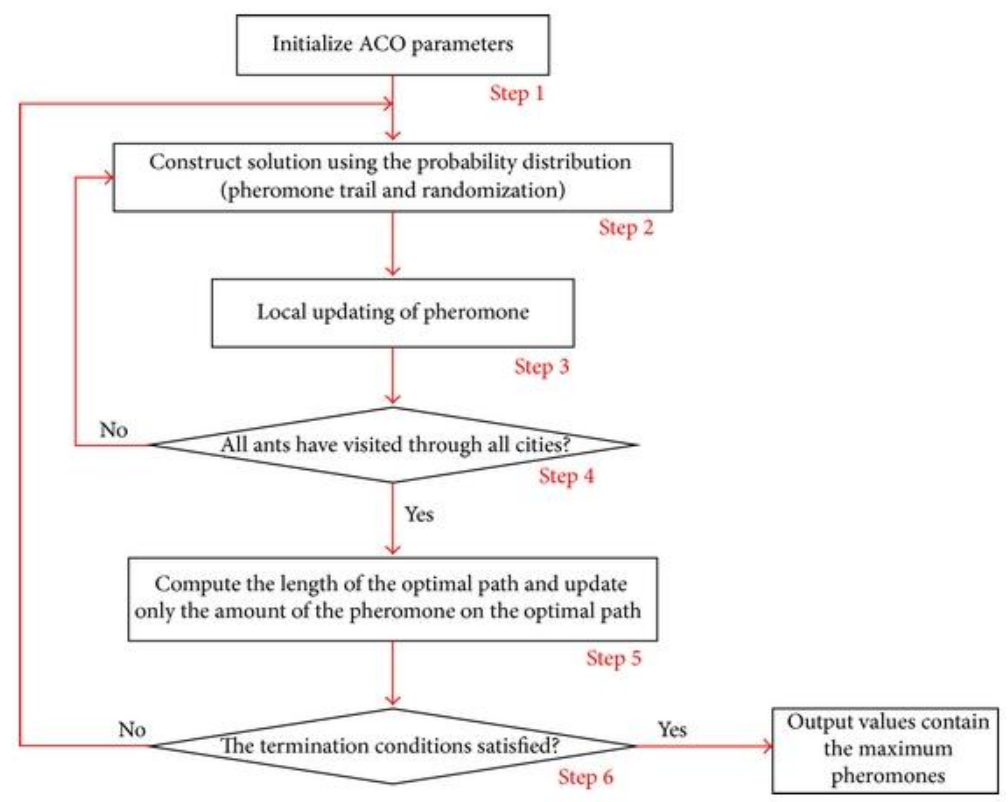

Fig. 2-Ant Colony Optimization Algorithm[5] 
- When an obstacle appears on the path of the ants.

- All the ant chain divides into two halves following the pheromone trails.

- Some will follow the shorter path and some will follow the longer one.

\subsection{Algorithm}

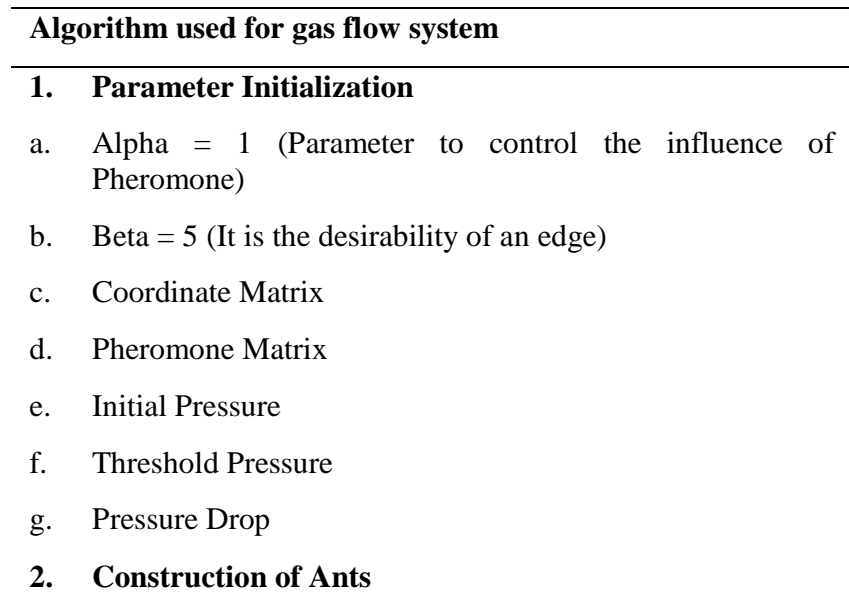

- Those ants who chose the shorter path will reconstitute around the shorter path.

- This will increase the pheromone amount around that path.

- Hence optimizing the path for them to follow.
a. Number of Ants = Number of Nodes
b. Each ant selects a city and performs its iteration.
3. Construct Solutions
a. Probability Distribution

\subsection{Output Screen}

COORDINATE MATRIX

$\begin{array}{lll}0 & 7 & 0 \\ 2 & 1 & 0 \\ 5 & 12 & 0 \\ 7 & 4 & 0 \\ 11 & 3 & 0 \\ 15 & 8 & 0\end{array}$

DISTANCE MATRIX

$\begin{array}{lllllll}0.000000 & 6.324555 & 7.071068 & 7.615773 & 11.704700 & 15.033296 \\ 6.324555 & 0.000000 & 11.401754 & 5.830952 & 9.219544 & 14.764823 \\ 7.071068 & 11.401754 & 0.000000 & 8.246211 & 10.816654 & 10.770330 \\ 7.615773 & 5.830952 & 8.246211 & 0.000000 & 4.123106 & 8.944272 \\ 11.704700 & 9.219544 & 10.816654 & 4.123106 & 0.000000 & 6.403124 \\ 15.033296 & 14.764823 & 10.770330 & 8.944272 & 6.403124 & 0.000000\end{array}$

ETA MATRIX
0.000000
0.158114
0.141421
0.131306
0.085436
0.066519
0.158114
0.000000
0.087706
0.171499
0.108465
0.067729
0.141421
0.087706
0.000000
0.121268
0.092450
0.092848
0.131306
0.171499
0.121268
0.000000
0.242536
0.111803
0.085436
0.108465
0.092450
0.242536
0.000000
0.156174
0.066519
0.067729
0.092848
0.111803
0.156174
0.000000 
PHEROMONE MATRIX
0.000000
1.000000
1.000000
1.000000
1.000000
1.000000
1.000000
0.000000
1.000000
1.000000
1.000000
1.000000
1.000000
1.000000
0.000000
1.000000
1.000000
1.000000
1.000000
1.000000
1.000000
0.000000
1.000000
1.000000
1.000000
1.000000
1.000000
1.000000
0.000000
1.000000
1.000000
1.000000
1.000000
1.000000
1.000000
0.000000

PROBABILITY MATRIX
0.000000
0.036338
0.020801
0.014353
0.001674
0.000479
0.0363380 .000000
0.001908
0.054553
0.005520
0.000524
0.020801
0.001908
0.000000
0.009644
0.002483
0.002537
$0.014353 \quad 0.054553$
0.009644
0.000000
0.308598
0.006424
$0.001674 \quad 0.005520$
0.002483
0.308598
0.000000
0.034163
0.000479
0.000524
0.002537
0.006424
0.034163
0.000000

ANT B: A-->B-->D-->E-->F-->C-->

Distance : 33.45 units

ANT C: A-->C-->D-->E-->F-->B-->

Distance : 40.61 units

ANT D: A-->D-->E-->F-->C-->B-->

Distance : 40.31 units

ANT E : A $-->\mathrm{E}-->\mathrm{D}-->\mathrm{B}-->\mathrm{C}-->\mathrm{F}-->$

ANT $\mathrm{E}: \mathrm{A}-->\mathrm{E}-->\mathrm{D}-->\mathrm{B}-->\mathrm{C}-->\mathrm{F}-->$

Distance : 43.83 units

ANT $\mathrm{F}: \mathrm{A}-->\mathrm{F}-->\mathrm{E}-->\mathrm{D}-->\mathrm{B}-->\mathrm{C}-->$

Distance : 42.79 units

Shortest distance: 33.452067 units

Initial pressure is assumed to be: $1000 \mathrm{psi}$

Threshold pressure is assumed to be: 600 psi

Pressure drop per unit distance is assumed to be: 100 psi

Number of Booster Pumps required to transmit Gas: 6 


\section{CONCLUSION AND FUTURE SCOPE}

The algorithm implemented in this project aims on optimization of gas transmission pipeline. The implementation of this project assists the user to minimize the use of resources like booster pumps. This algorithm produces results only for $(\mathrm{X}, \mathrm{Y})$ coordinates only and is currently insufficient for $\mathrm{Z}$ coordinate. Currently this project works as an implementation of Ant Colony Optimization Algorithm for optimized transmission of Gas and can only be implemented on 2-D plane. In future, the project will be capable enough to work on 3 dimensions. This project gives the best route on which the pipeline is to be laid.

\section{REFERENCES}

[1] Baeza, Daniel \& Ihle, Christian \& Ortiz, Julian. (2017). A comparison between ACO and Dijkstra algorithms for optimal ore concentrate pipeline routing. Journal of Cleaner Production. 144. 149-160. 10.1016/j.jclepro.2016.12.084.

[2] Soeiro Ferreira, José \& Vidal, René. (1984). Optimization of a pump-pipe system by dynamic programming. Engineering Optimization - ENG OPTIMIZ. 7. 241-251. 10.1080/03052158408960641

[3] Joy deep Kundu. Ant colony optimization problem to solve travelling salesman problem.

[4] "SDLC - RAD Model", Tutorials Point (2016), website:https://www.tutorialspoint.com/sdlc/sdlc_rad_m odel.htm\#

[5] Won Hyung, Flowchart-of-ACO-Ant-ColonyOptimization, AS:328302964297731@1455284934219, website:
https://www.researchgate.net/profile/Won_Hyung/public ation/274620390/figure/fig9/AS:328302964297731@14 55284934219/Flowchart-of-ACO-Ant-ColonyOptimization.png

[6] Bora, Neha, Jigyasha Arora, and Nitin Arora. "Ant colony based optimization algorithm for the Mobile ad hoc Network Routing Problems based on SMR Protocol (ACO-SMR)." International Journal of Application or Innovation in Engineering \& Management, Vol. 2, 6, June 2013.

[7] Lakshmi, M., Kaushik, P. K., \& Arora, N. (2013). Novel Approximation Algorithm for Calculating Maximum Flow in a Graph. International Journal of Computer Applications, 74(3).

[8] Kumar, Ashwani, Surinder Pal Singh, and Nitin Arora. "A New Technique for Finding Min-Cut Tree." International Journal of Computer Applications 69, no. 20 (2013).

[9] Kumar, Ashwani, Surinder Pal Singh, and Nitin Arora. "A New Technique for Finding Min-Cut Tree." International Journal of Computer Applications 69, no. 20 (2013).

[10] Arora, Nitin, Pradeep Kumar Kaushik, and Surinder Pal Singh. "A Survey on Methods for finding Min-Cut Tree." International Journal of Computer Applications 66, no. 23 (2013).

[11] Bora, Neha, Swati Arora, and Nitin Arora. "An Efficient Algorithm for Calculating Maximum Bipartite matching in a Graph." International Journal of Advanced Computer Research 3, no. 2 (2013): 193. 